\title{
The kinematic properties of isolated elliptical galaxies
}

\author{
George K. T. $\mathrm{Hau}^{1} \dagger$, Duncan A. Forbes ${ }^{2}$ and Fatma Reda ${ }^{2,3}$ \\ ${ }^{1}$ European Southern Observatory, Karl-Schwarzschild-Str. 2, D-85748, Garching-bei-München, \\ Germany email: ghau@eso.org \\ ${ }^{2}$ Centre for Astrophysics and Supercomputing, Swinburne University of Technology, \\ Hawthorn, VIC 3122, Australia \\ ${ }^{3}$ Astronomy Department, National Research Institute of Astronomy and Geophysics, Helwan, \\ Cairo 11421, Egypt
}

\begin{abstract}
Ellipticals in very low density environments are extremely rare but hold important clues about galaxy formation and evolution. We present results of an investigation of their internal dynamics. We observe a dichotomy in kinematic properties similar to that in normal ellipticals. We compare the positions of isolated ellipticals with respect to the Fundamental Plane of normal ellipticals and find that they fall onto it, with the exception of NGC 2865. This shows that isolated ellipticals are structurally similar to normal ellipticals, and do not have very young ages, although some have signs of past mergers or interactions.
\end{abstract}

\section{Introduction}

It is relatively easy to study large numbers of cluster galaxies because a single CCD image or spectral mask may contain hundreds of galaxies. For low density environments, the same CCD may contain only one or two galaxies. Consequently, our knowledge of the nearby universe is often directly proportional to the environmental density. Very isolated galaxies (sometimes called the 'extreme field') are consequently the least studied. Although poorly studied, this extreme in the density range of galaxy environments offers crucial 'leverage' to tackle several outstanding issues in galaxy evolution. For example, the current hierarchical models for galaxy formation predict that ellipticals in low density environments have younger mean stellar populations and lower $[\mathrm{Mg} / \mathrm{Fe}]$ ratios than their cluster counterparts (e.g. Kauffmann \& Charlot 1998). Isolated ellipticals are thought to have formed in relatively recent mergers of spiral galaxy pairs. If so, we might expect a high incidence of Kinematically Distinct/Decoupled Cores (KDC). If formed in the last few Gyrs, they should deviate from the Fundamental Plane of normal ellipticals (Forbes, Ponman \& Brown 1998).

\section{Sample}

Reda et al. (2004) defined a new sample of isolated galaxies. Early type galaxies outside of groups and clusters, with $\mathrm{B}_{T} \leqslant 14$, were selected from the LEDA database. These were further restricted to have no neighbours within $700 \mathrm{~km} / \mathrm{s}$ in velocity, $0.67 \mathrm{Mpc}$ on the sky and $2 \mathrm{~B}$ band magnitudes. Finally a visual check using the DSS was carried out. This resulted in a sample of 36 very isolated $\mathrm{E} / \mathrm{S} 0$ galaxies. Wide-field imaging results of 10

$\dagger$ Current address: Department of Physics, Durham University, South Road, Durham DH1 3LE, UK 


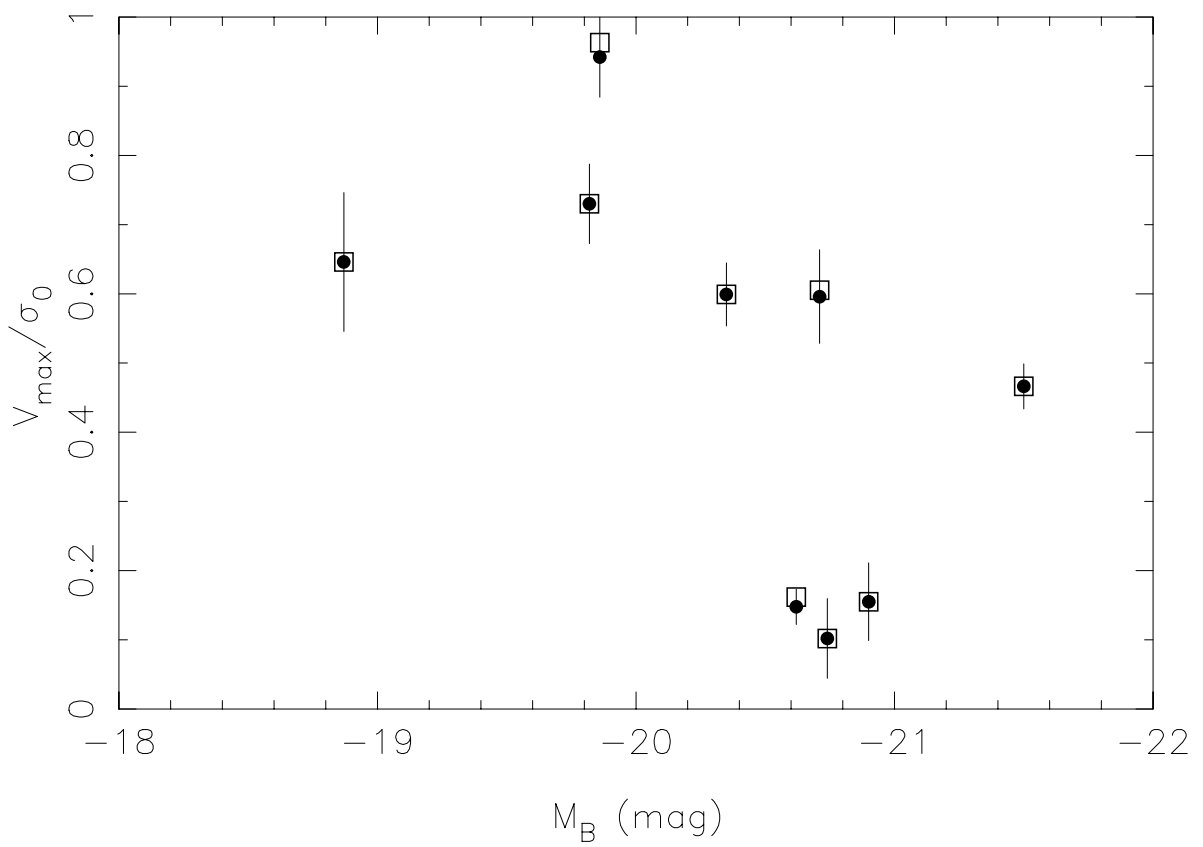

Figure 1. Measured $V_{\max } / \sigma_{0}$ for the sample. The solid dots and open squares represent the values before and after correction for the small misalignment between the adopted slit position angle and the actual position angle of the major axis, respectively.

galaxies in this sample are reported in Reda et al. (2004). Like Kuntschner et al. (2002), Reda et al. found several isolated galaxies with signs of recent morphological disturbance.

\section{Results}

A subsample of 9 isolated ellipticals were observed using EFOSC2 at the ESO $3.6 \mathrm{~m}$. The Line-of-Sight Velocity Distributions (LOSVDs) were parameterised by velocity $v$, velocity dispersion $\sigma$, and Gauss-Hermite moments $h_{3}$ and $h_{4}$ (van der Marel \& Franx 1993).

In Fig. 1 we plot the measured $V_{\max } / \sigma_{0}$ for the sample, where $V_{\max }$ is the maximum rotational velocity and $\sigma_{0}$ is the central velocity dispersion. A kinematic dichotomy can be seen: faint ellipticals are rotationally supported whereas for $M_{B}<-20$ both populations exist. This dichotomy is a well known property of normal ellipticals (Bender 1988, Bender et al. 1989). In our sample, the number of rotationally supported ellipticals outnumber the dispersion supported ones by 2 to 1 .

In Fig. 2 we plot the positions of the isolated galaxies with respect to the Fundamental Plane, seen edge-on, of normal ellipticals (Burstein et al. 1987, de la Rosa, de Carvalho \& Zepf 2001). Isolated ellipticals fall onto it, with the exception of NGC 2865, a 1.2 Gyr old merger remnant with a KDC (Hau et al. 1999). This suggests that the majority of isolated ellipticals are structurally similar to normal ellipticals, and that they do not have very young ages ( 1.2 Gyr, Forbes, Ponman \& Brown 1988).

We found that 4 out of 9 galaxies in our sample have KDCs. Their occurrence is slightly higher than that for the overall elliptical galaxy population (Jedrzejewski \& Schechter 1988, Bender 1990, 1996), although the classification can be subjective. Several galaxies in our sample have asymmetric kinematics which may indicate recent disturbances, and/or localised dust obscuration. There are also a few cases of solid-body rotation. Thus, 


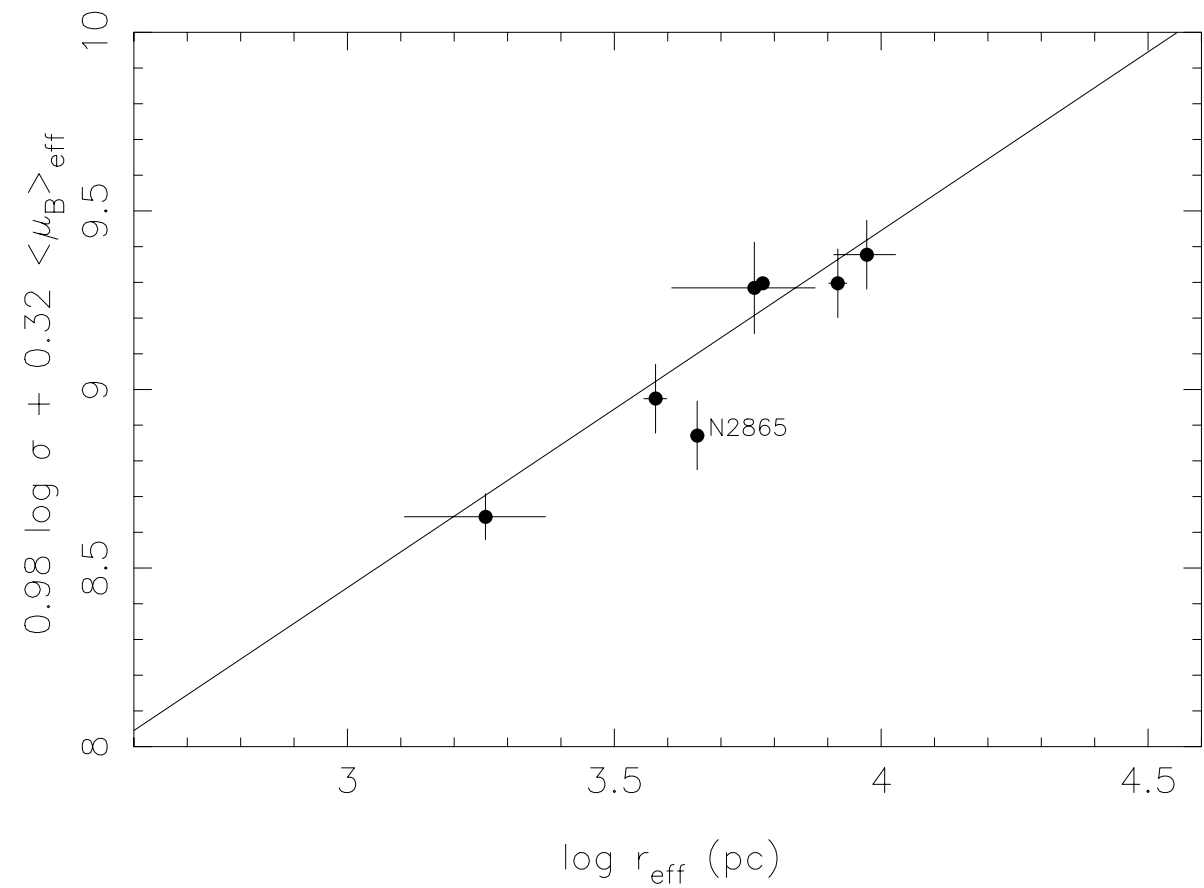

Figure 2. The positions of the isolated galaxies with respect to the Fundamental Plane of normal ellipticals $\left(\log r_{\text {eff }}=0.98 \log \sigma+0.32\left\langle\mu_{B}\right\rangle_{\text {eff }}-5.57\right)$, corrected to a Hubble constant of $75 \mathrm{~km} \mathrm{~s}^{-1} \mathrm{Mpc}^{-1}$. This relationship was calculated by de la Rosa, de Carvalho \& Zepf (2001) based on a subset of galaxies in Burstein et al. (1987).

although the Fundamental Plane result does not support very young ages, a significant fraction of isolated ellipticals have signs of past mergers or interactions, supporting the finding of morphological disturbances (Kuntschner et al. 2002, Reda et al. 2004)

Details on the Fundamental Plane study and on the kinematics of individual galaxies shall be present in forthcoming papers (Reda, Hau \& Forbes in preparation, Hau \& Forbes in preparation).

\section{References}

Bender R., 1988, A\&A, 193, L7

Bender R., Surma P., Doebereiner S., Moellenhoff C., Madejsky R., 1989, A\&A, 217, 35

Bender R., 1990, A\&A, 229, 441

Bender R., 1996, IAUS, 171, 181

Burstein D., Davies R. L., Dressler A., Faber S. M., Stone R. P. S., Lynden-Bell D., Terlevich R. J., Wegner G., 1987, ApJS, 64, 601

de la Rosa I. G., de Carvalho R. R., Zepf S. E., 2001, AJ, 122, 93

Forbes D. A., Ponman T. J., Brown R. J. N., 1998, ApJ, 508, L43

Hau G. K. T., Carter D., Balcells M., 1999, MNRAS, 306, 437

Jedrzejewski R., Schechter P. L., 1988, ApJ, 330, L87

Kauffmann G., Charlot S., 1998, MNRAS, 294, 705

Kuntschner H., Smith R. J., Colless M., Davies R. L., Kaldare R., Vazdekis A., 2002, MNRAS, 337,172

Reda F. M., Forbes D. A., Beasley M. A., O’Sullivan E. J., Goudfrooij P., MNRAS submitted van der Marel R. P., Franx M., 1993, ApJ, 407, 525 